\title{
EL PATRIMONIO EPIGRÁFICO GRIEGO DE LA COMUNIDAD DE MADRID*
}

\author{
Ma Luisa del Barrio Vega - Paloma Guijarro Ruano \\ Universidad Complutense de Madrid \\ marisaba@ucm.es - palguija@ucm.es
}

\begin{abstract}
RESUMEN
El presente trabajo tiene por objetivo presentar una visión general de las inscripciones griegas que actualmente se conservan en la Comunidad de Madrid. A diferencia del criterio seguido en IGEP (Inscripciones griegas de España y Portugah), que solo recoge el material epigráfico inscrito en la península ibérica y Baleares o que ha llegado en época antigua, aquí nos ocupamos también de las inscripciones que han sido introducidas en época moderna, principalmente a través de donaciones o adquisiciones, con independencia de cuál sea su procedencia, la fecha de su introducción en la península o el tipo de soporte en que se encuentran.
\end{abstract}

PALABRAS CLAVE: inscripciones griegas, monedas griegas, vasos griegos, Comunidad de Madrid.

GREEK INSCRIPTIONS OF THE COMUNIDAD AUTÓNOMA OF MADRID

\section{ABSTRACT}

The objective of this work is to offer an overall picture of all the Greek inscriptions currently stored in the Comunidad of Madrid. In contrast with the ordering criteria displayed in IGEP (Inscripciones griegas de España y Portugal), which only collects epigraphic material inscribed in the Iberian Peninsula (or inscribed outside but arrived there in ancient times), we also deal with Greek inscriptions introduced in the Peninsula in modern times, regardless their origin, the date of their introduction in the country and the typology of the epigraphical objects. These documents were manly purchased through donations and acquisitions.

KEYwORDS: Greek inscriptions, Greek coins, Greek vases, Comunidad of Madrid.

\section{LA COMUNIDAD DE MADRID Y EL PATRIMONIO EPIGRÁFICO GRIEGO}

No son muchas las inscripciones griegas conservadas en las colecciones y museos de la Comunidad de Madrid'. La mayoría de ellas se encuentran en el Museo Arqueológico Nacional (MAN), en el Museo del Prado, en el Servicio de Arqueología del Ayuntamiento de Alcalá de Henares y en el Gabinete de Antigüedades de la Real Academia de la Historia, a los que hay que añadir el Museo de la Casa de la Moneda 
para las monedas griegas. Los epígrafes pertenecen a diversas épocas y se encuentran en diferentes soportes: grafitos comerciales, inscripciones en vasos cerámicos, dedicaciones, monedas e incluso una fíbula de oro.

El corpus más reciente y exhaustivo de inscripciones griegas antiguas de la península ibérica, Inscripciones griegas de España y Portugal (de Hoz, 2014 = IGEP), comprende alrededor de 600 epígrafes $^{2}$. La distribución geográfica y cronológica de las inscripciones es bastante desigual. En efecto, mientras que de algunas regiones conservamos un número relativamente numeroso de inscripciones griegas, las conservadas en otras comunidades son bastante escasas. Es el caso de la Comunidad de Madrid (CAM), que en el corpus mencionado cuenta con menos de veinte ejemplares. Además, solamente tres de ellos proceden de la misma CAM, los tres encontrados en Complutum (Alcalá de Henares) (cf. \$1). El resto de los epígrafes recogidos en IGEP proviene de yacimientos de otros lugares de España (cf. infra \$\$2-3). Sin embargo, como indica la autora en la introducción, IGEP no recoge todas las inscripciones griegas conservadas en la península y Baleares, sino solo aquellas que han sido inscritas aquí o que han llegado como consecuencia del contacto directo con griegos y orientales. Por tanto, no incluye los epígrafes antiguos procedentes de donaciones y adquisiciones posteriores de época moderna, como son la mayoría de las inscripciones pintadas en los vasos cerámicos del MAN o las dedicaciones que se encuentran en el Museo del Prado.

El presente trabajo pretende, por tanto, ofrecer una visión general de las inscripciones escritas en griego conservadas en la Comunidad de Madrid, incluyendo aquellas que han llegado en época moderna y completando de este modo trabajos y estudios anteriores.

Sirva este pequeño estudio de modesto homenaje a nuestro colega y amigo el profesor Ángel Martínez Fernández, gran conocedor de la epigrafía griega.

\section{LAS TRES INSCRIPCIONES DE ALCALÁ DE HENARES}

Como se ha dicho supra, las tres inscripciones depositadas en el Servicio de Arqueología del Ayuntamiento de Alcalá de Henares son los únicos epígrafes griegos, hasta donde sabemos, hallados en suelo de la CAM. Los tres se encuentran grabados

* Este trabajo se enmarca dentro de los proyectos de investigación FFI2017-82590-C2-1-P y TEAPIMEG GR35/10-A-93075.

${ }^{1}$ Existen algunas bases de datos disponibles on-line para la búsqueda del material epigráfico griego en la península Ibérica. A tal efecto puede consultarse la web del proyecto Iberia Graeca https://iberiagraeca.net/base/index.php o, con un carácter más general, la Red Digital de Colecciones de Museos de España CER.ES http://ceres.mcu.es/pages/SimpleSearch?index=true.

${ }^{2}$ El corpus comprende las inscripciones griegas de España y Portugal, más las de las islas Baleares. Véanse las reseñas de Ramírez Sánchez (2015), Sarrazanas (2016) y Guijarro Ruano (2016). 
en fragmentos cerámicos. Dos contienen un nombre incompleto y el tercero puede ser interpretado de diferentes maneras.

Entre los siglos I-II d.C. se fecha un fragmento de cuenco (IGEP 452; HEpigr. 7, 1997 [2001], no 405c) con el nombre fragmentario A $\rho \tau \varepsilon i ́ \mu \omega v[--]$, en lugar de la forma esperable A $А \tau \varepsilon \mu-$, que puede estar en nominativo, dativo o, más probablemente, en genitivo ('A $\rho \tau \varepsilon i ́ \mu \omega \vee o \varsigma)$. Basándose en diversas formas citadas por Gignac (1976: 256) en su gramática de los papiros, la autora de IGEP (cf. p. 17)

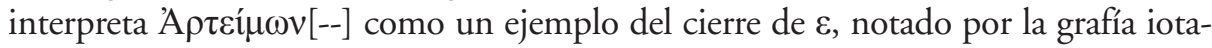
cista $<$ EI $>$. Según esta interpretación, se trataría de una simple confusión gráfica entre $<\varepsilon>\mathrm{y}\langle\varepsilon l>(=[\mathrm{i}])$ debida a la pérdida de oposición de la cantidad vocálica y a los diferentes procesos de iotacismo que ya han tenido lugar en este periodo 3 . Pero la cuestión es más compleja. En primer lugar, no parece que haya en Hispania otros ejemplos semejantes de este supuesto cierre de $\varepsilon$. Segundo, como ya se indica en $I G E P$ (p. 504), A $\rho \tau \hat{\varepsilon} \mu \omega v$ es un antropónimo muy común en el mundo griego y especialmente en Asia Menor. Sin embargo, hay que señalar que la variante con $\varepsilon 1$ parece ser exclusiva de esta última región, de donde proceden casi todos los ejemplos atestiguados, en su inmensa mayoría pertenecientes a los antropónimos A $\rho \tau \varepsilon \mu \alpha \varsigma$, A

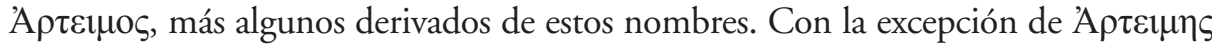
(Robert, Sinuri 5, Caria, siglos V-IV a.C.), la mayoría de los ejemplos es posterior al siglo I a.C., siendo estos especialmente numerosos en época imperial ${ }^{4}$.

Son varios los estudiosos que se han ocupado de los nombres tipo A $А \tau \varepsilon \mu^{\circ}$ / A $\rho \tau \mu^{\circ}$ y los han relacionado con antropónimos anatolios. ${ }^{5}$. Por lo general, consideran que las variantes $A \rho \tau \varepsilon \mu \mu^{\circ}$ y $A \rho \tau \mu \mu^{\circ}$ son producto de la helenización de diversos nombres indígenas (cf. lidio Artimu- y cario Artmi-, entre otros), bajo la influencia de los antropónimos griegos formados con el nombre de la diosa 'A $\rho \tau \varepsilon \mu 1 \varsigma^{6}$. Como indica L. Robert $(1963,70)$, el parecido entre los nombres indígenas y estos nombres

${ }^{3}$ Cf. de Hoz en IGEP p. 17: «El cambio $\varepsilon>\varepsilon 1$ debido a la pronunciación cerrada de la vocal

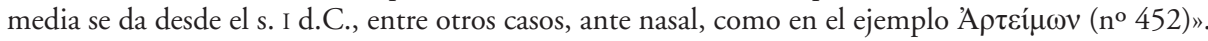

${ }^{4}$ De ser correcta la datación propuesta por Robert en su edición de 1945, la fecha temprana del ejemplo de Sinuri confirmaría que $\varepsilon 1$ por $\varepsilon$ en estas formas no es un mero hecho fonético.

${ }^{5}$ Cf. Robert (1963: 70); Zgusta (1964: 99-102); Brixhe (1991: 77-78); Melchert (2013: 37); Vernet (2012-2014).

${ }^{6}$ Vernet (2012-2014) atribuye diferente etimología a las dos variantes, Artimas y Arteimas (irania y epicórica, respectivamente), subestimando, en nuestra opinión, la influencia del griego, cuyos datos, por otra parte, analiza erróneamente. En efecto, la autora no parece tener en cuenta en su análisis la fecha de los ejemplos griegos ni la posible intervención de los procesos de iotacismo propios del griego tardío, que justificaría el empleo de $<\mathrm{EI}>=$ /i/. Por otra parte, explica la forma griega A $\rho \tau \varepsilon \mu \alpha \varsigma$ como resultado de una metátesis de la $l$ a partir de la forma *Artemi-a-s, proceso que, según Vernet (p. 106), es frecuente en griego en los grupos en que la $i(s i c)$ iba precedida de una líquida o nasal: cita

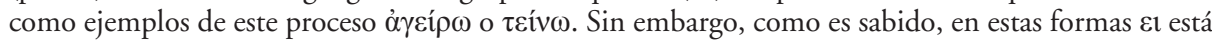
representando una /e:/ procedente de un alargamiento compensatorio y no un diptongo, que solo se da cuando ${ }^{*} r y{ }^{*} n y$ van precedidos de una vocal de timbre $a$ y $o$. 
griegos habría favorecido la gran difusión de estos últimos en esta zona ${ }^{7}$. A este respecto, no podemos dejar de citar dos pasajes de Brixhe (1991) a propósito de la helenización de los nombres indígenas de Asia Menor: «Lors de l'adoption du grec, le patrimoine onomastique indigène survit au-delà de la période de bilinguisme, naturellement au prix d'une adaptation phonologique et morphologique au grec» (p. 68); «L'hellénisation d'une forme indigène procède donc souvent de son identification à un radical homophone ou quasi homophone, avec (dans ce dernier cas) modification phonétique» (p. 79).

Así pues, los nombres con vocalismo A $\rho \tau \varepsilon u \mu-$, A $\rho \tau \iota \mu-$ atestiguados en Asia Menor deben interpretarse como nombres indígenas helenizados, frente a los propiamente griegos que presentan $A \rho \tau \varepsilon \mu^{\circ}$. Volviendo al A $A \tau \varepsilon i ́ \mu \omega v$ del cuenco hallado en Alcalá, aunque carecemos de otros indicios que permitan determinar el origen del portador de este nombre, resulta tentador considerar que se trata de un individuo (¿griego?) procedente de Asia Menor, quizás un esclavo de la ciudad roma-

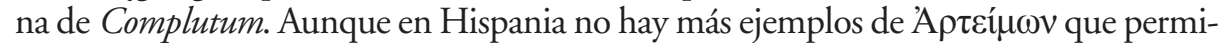

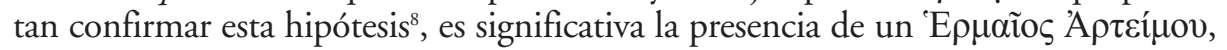
originario de Termessos, en un largo epigrama funerario procedente de Roma de la misma fecha que el cuenco de Alcalá (IGUR III 1204, s. I-II d.C.).

De la misma época es un fragmento de copa (IGEP 453; HEpigr. 7, 1997

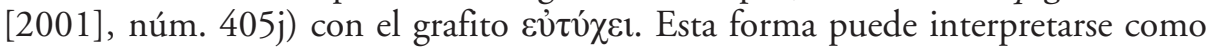

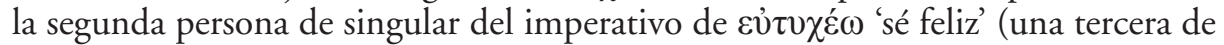

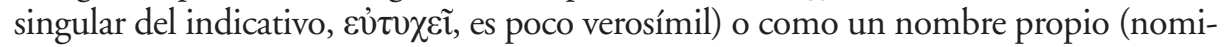
nativo Ev̉ $u ́ \chi\rceil \varsigma)$, bien en dativo indicando el destinatario del vaso ('para Eutiques'), bien, como es más frecuente en los vasos, en genitivo indicando el propietario ('de

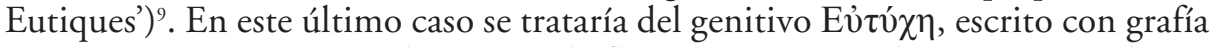
iotacista $(<\mathrm{EI}>$ por $<\mathrm{H}>)$, de un tipo de flexión ya atestiguado en griego tardío en

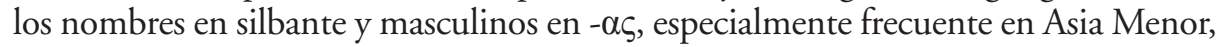
y que se va a convertir en regular en el griego posterior y moderno: nom. $-\eta \varsigma$ y $-\alpha \varsigma$, acusativo y genitivo $-\eta$ y $-\alpha^{10}$.

Los nombres formados con Ev̉ $v \chi^{\circ}$ están bien atestiguados en todo el territorio de habla griega, siendo especialmente frecuentes en la zona de Asia Menor (cf.

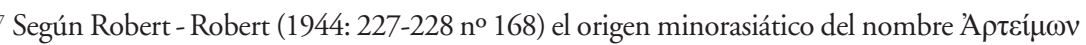
cuadraría bien con la procedencia y fecha de los ejemplos. Sobre la alternancia A $\rho \tau \varepsilon \mu^{\circ} / \mathrm{A} \rho \tau \mu^{\circ}$, véase Brixhe (1991: 78).

${ }^{8}$ Según el índice onomástico de $I G E P$, en la península ibérica solo existe otro ejemplo derivado

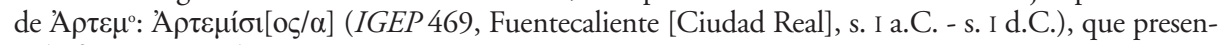
ta la forma esperada.

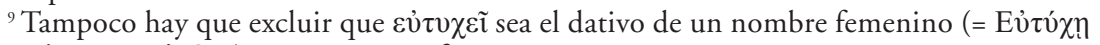

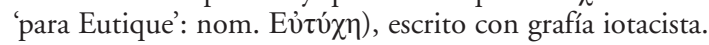

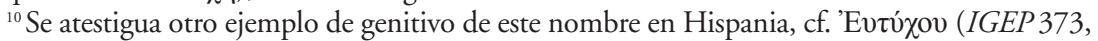
Majadaiglesia, Córdoba, ¿̇s. I d.C.?); de estar en genitivo (y no en acusativo, cf. IGEP p. 400), puede

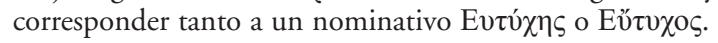


$L G P N \vee A, v B, v C)$. La presencia de estos nombres en Italia e Hispania podría deber-

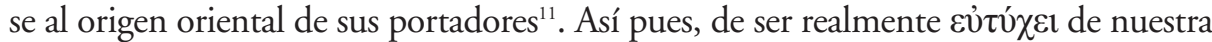
copa un antropónimo, podríamos estar de nuevo ante un individuo de procedencia oriental.

El tercer fragmento (IGEP 454; HEpigr. 4, 1994, no 525; siglos III-IV d.C.) lleva inscrito otro nombre, también incompleto, $\Phi \omega \tau i ́ \omega v[--]$, el cual, como en el caso

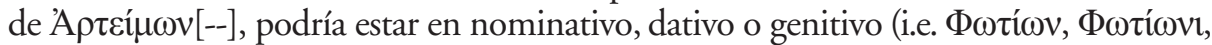

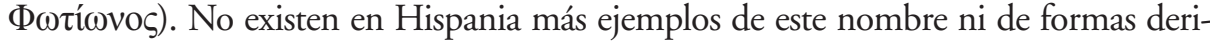
vadas de ${ }^{\circ} \varphi \omega \tau^{\circ}$ luz' (tampoco de su equivalente - $\left.\varphi \alpha ́ \omega v /-\varphi \tilde{\omega} v\right)$. Los nombres con esta

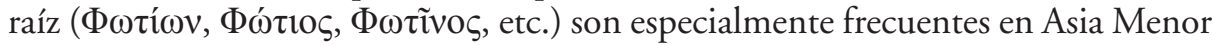
y otras zonas de Oriente. Como en el caso de A $\rho \tau \varepsilon i ́ \mu \omega v[--]$, uno se sentiría tentado a identificar $\Phi \omega \tau i ́ \omega v[--]$ con una persona de condición servil procedente de Asia Menor, pero, más allá de estos nombres, carecemos de argumentos sólidos que apunten en esta dirección.

\section{LAS INSCRIPCIONES GRIEGAS DEL GABINETE DE ANTIGÜEDADES DE LA REAL ACADEMIA DE LA HISTORIA}

En este apartado nos limitaremos a citar IGEP 66 (no de inv. 2000/10/1), ya que, como indica su autora, las otras tres inscripciones incluidas en el corpus (A III 1.2, A III 2.2, A III 2.3) son falsas. IGEP 66 es un grafito inscrito en un skyphos ático del primer tercio del siglo IV a.C., con una indicación numérica comercial del mismo tipo que algunas de las conservadas en el MAN (cf. infra \$3.1). Fue encontrado en Ampurias (M. Almagro-Gorbea, Epigrafia prerromana 2003, 91-92, no 7, con fotos; SEG 54.996, HEpigr. 13, 2003-4 [2007], 132, no 334). En la parte central izquierda

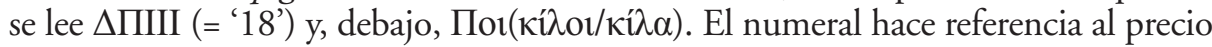
o al número de piezas del lote.

\section{LAS INSCRIPCIONES GRIEGAS DEL MUSEO ARQUEOLÓGICO NACIONAL}

\subsection{Grafitos COMERCiales.}

Casi todos los grafitos de carácter comercial son numerales, algunos de ellos muy breves, y proceden de diferentes yacimientos arqueológicos.

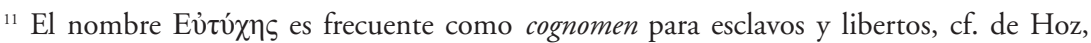
IGEP 454, con bibliografía anterior. Para otras formas del antropónimo en Hispania, cf. IGEP nn. 220,

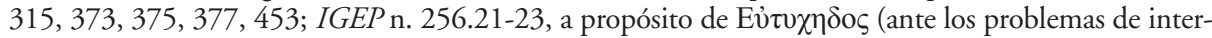
pretación que presenta esta forma, consideramos preferible no acentuarla). 
El grafito más antiguo está inscrito en un ánfora de aceite del siglo VII a.C. procedente de Toscanos (Torre del Mar, Vélez-Málaga) (MAN inv. TM 67/152/1018; IGEP 329; SEG 51.1468.2): [- - - ] tario en genitivo. Del último cuarto del siglo V a.C. es otro grafito comercial inscrito en una lucerna hallada en el yacimiento de Puig des Molins (Ibiza) (MAN inv. 36364; 1923/60/740; IGEP 499): $\mathrm{HH}^{\top} \Delta \Delta \Delta$. Probablemente la cantidad indicada $(200+50+30=280)$ hace referencia al número de lucernas de que constaba el lote.

Algo posteriores (siglos V-IV a.C.) son otros dos grafitos, muy breves, inscritos en sendos fragmentos de pie de cratera, ambos procedentes del yacimiento del Cerro del Real (Granada). Uno de ellos (MAN inv. 1979/70/GAL/342; IGEP 320) consta de una sola letra, $\Delta\left(={ }^{\prime} 10\right.$ '). El otro (MAN inv. 1979/70/258; IGEP 319), consta de dos: $\Delta \Delta[---](=' 20$ ' + ¿?).

Del siglo IV a.C. es un grafito inscrito en una cratera de figuras rojas procedente de Peal de Becerro (Jaén) con el numeral $\Delta \Delta \Pi$ ('15'), que seguramente indica el número de objetos más pequeños contenidos en la cratera (MAN inv. 1986/149/209 = 32.713; IGEP 321). Del mismo siglo, pero algo más extenso es el grafito que se lee en la base de una cratera de campana de figuras rojas del siglo IV a.C., procedente del yacimiento de Villaricos, Almería (MAN inv. 84/152/2; IGEP 313; SEG 51.1468.11):

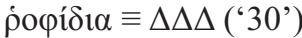

$$
\begin{aligned}
& \text { ПII ('7') }
\end{aligned}
$$

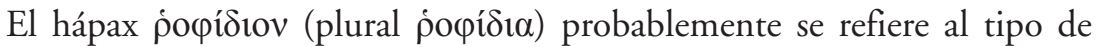
vasos transportados en la cratera en la que se encuentra inscrito el grafito. Este tér-

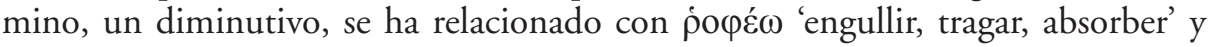
фó $\varphi \eta \mu \alpha$ 'lo que se engulle o traga' o 'especie de papilla', una raíz que encaja perfectamente con el objeto (cf. de Hoz en IGEP p. 322) ${ }^{12}$. Los numerales deben de indicar el número de poóídı transportados y el precio de estos o quizás el de la cratera.

\subsection{INSCRIPCIONES EN OTROS MATERIALES}

Conservamos dos epígrafes en piedra, ambos tardíos. Uno de ellos, de época imperial y procedente de Baria (Almería), se encuentra inscrito en el pedestal de una estatua de la musa Clío que se ha perdido (MAN inv. 1907/32/68; IGEP 314):

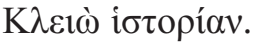

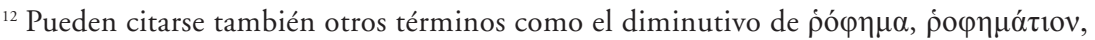
las formas jonias equivalentes en $\dot{v} \varphi$-, así como el término tò po «item in list of kitchen vessels, Lang, Ath.Agora XXI B12 (pl. IV/III BC»). 
El otro es un fragmento de una tumba de mármol del siglo VI d.C. hallado en Mérida (MAN inv. 57776; IGEP 411; Hübner, $I H C$ no 348; Ramírez - Mateos $2000 \mathrm{n}^{\circ}$ 184; HEpigr. 9, 1999 (2003), no 225; SEG 52.1005). El texto del grafito se ha conservado en estado fragmentario y es difícil de interpretar. En la parte que se conserva se ve lo siguiente (cf. fotografía del calco en $I H C 348$ y de la inscripción en IGEP 411):

\section{† NE \\ ]EN MHN \\ ]TPITE + $\Psi$}

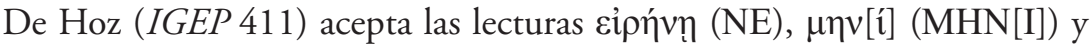
$\tau$ qítn (TPITE) de la mayoría de los editores anteriores y presenta el siguiente texto:

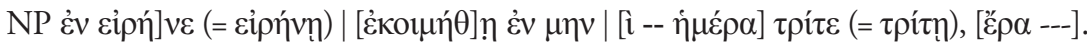

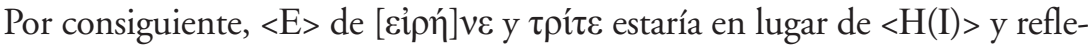
jaría la probable pronunciación no iotacista de $\eta$ en algunas zonas de oriente (Siria y Palestina), como ocurre también en otras zonas periféricas (la región del Ponto) ${ }^{13}$. Esto es lo que también parece mostrar la confusión entre $<\mathrm{E}\rangle \mathrm{y}\langle\mathrm{H}\rangle$ en una serie de epitafios de individuos orientales encontrados en occidente (Sicilia, Galia, Hispania misma), frente al iotacismo más extendido ${ }^{14}$. De hecho, contamos con otros ejemplos de confusión entre $<\mathrm{E}>\mathrm{y}<\mathrm{H}>$ en Hispania, por ejemplo, en IGEP 375 a y b

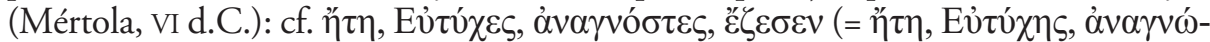
$\sigma \tau \eta \varsigma$, , $̌ \zeta \eta \sigma \varepsilon v)$; en la misma inscripción también se dan casos de confusión de $\langle\mathrm{O}>$

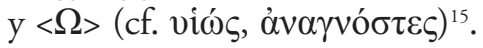

Mencionemos, por último, una inscripción cristiana sobre una fíbula de oro de fines del siglo VI d.C., grabada encima y debajo de una escena que representa a los Reyes Magos ante la Virgen y Jesús niño. (MAN inv. 1963/56/1; IGEP 389). La fíbula, hallada en una tumba en Medellín, probablemente procede de algún lugar de oriente (Siria o Palestina).

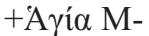

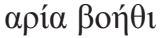

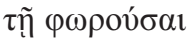

+ A $\mu$ ńv +

«Santa María, ayuda a la que lo lleva»

${ }^{13}$ Cf. Feissel (1982: 339); Brixhe (1987: 109).

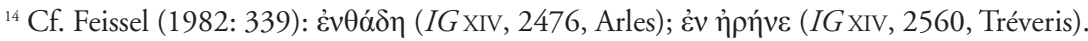

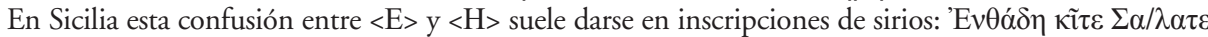

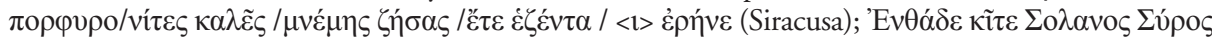
$\varepsilon \ddot{\mu} \mu \varepsilon \rho \circ \varsigma \alpha \alpha \lambda \tilde{\varepsilon} \varsigma \mu \nu \varepsilon \dot{\mu} \mu \varepsilon \varsigma$ (Siracusa).

${ }^{15}$ Pueden verse más ejemplos en IGEP p. 17. 
Por la fecha tardía de la inscripción no extraña la ausencia de la iota en el dativo $\tau \tilde{\eta}$ (en lugar de la grafía correcta $<\mathrm{THI}>$ ), que reflejaría la pérdida del segundo elemento del diptongo, ni la grafía iotacista en el imperativo $\beta$ oń $\theta \mathrm{t}(=\beta$ oń $\theta \varepsilon \imath)$, así

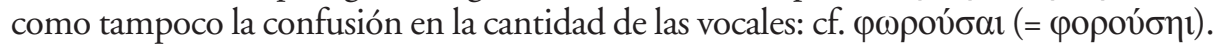
$-\alpha \iota$ en lugar de $-\eta \uparrow$ podría, además, reflejar la probable pronunciación no iotacista de $\eta$ (véase el comentario a la inscripción anterior). En efecto, $\varphi \omega \rho o v ́ \sigma \alpha \iota$ sería una

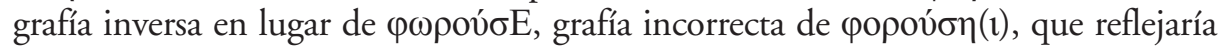
la pérdida del segundo elemento del diptongo, así como de la oposición de la canti-

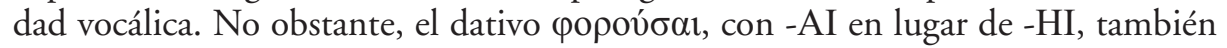
podría ser el resultado de la regularización del paradigma: la $-\alpha$ del nominativo y acusativo (conservada por ser breve) se habría extendido al genitivo y dativo, como ocurre en el griego moderno con los temas en - $\alpha$ larga mixtos (cf. nom. $\theta \alpha \dot{\lambda} \alpha \sigma \sigma \alpha$, gen. $\theta \alpha \dot{\lambda} \alpha \sigma \sigma \alpha \varsigma$ en lugar de $\theta \alpha \lambda \alpha ́ \sigma \sigma \eta \varsigma)$.

\section{LOS VASOS CERÁMICOS PINTADOS DEL MAN}

Como en el resto de los epígrafes, $I G E P$ solo recoge los vasos cerámicos pintados que han aparecido en algún yacimiento de la península o Baleares, bien fabricados aquí bien llegados en la Antigüedad como consecuencia del contacto directo con griegos y orientales. Por esta razón, de los vasos pintados conservados en el MAN dicho corpus sólo incluye una kylix hallada en Medellín y fechada en 560-550 a.C. (MAN inv. 1969/61/1, con muy buena foto en el catálogo on-line del museo; IGEP 388, SEG 51.1468.5; SEG 54.1001; HEpigr. 13, 2003-04 [2007], no 93). La inscrip-

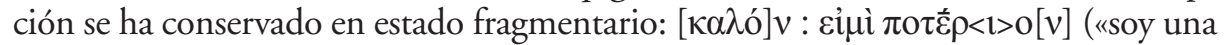
[hermosa] copa»). Según J. Curbera (HEpigr. 13, 2003-04 [2007], no 93) en el final de la primera palabra se podría leer quizás una $\langle\mathrm{Y}\rangle$, es decir, el final de la desinencia de genitivo del nombre del propietario de la copa («soy la copa de [---]»). Según de $\mathrm{Hoz}$ (IGEP 388), se lee claramente una $<\mathrm{N}>$. Como peculiaridad lingüística notemos que es uno de los textos epigráficos áticos en que, aunque escritos en alfabeto epicórico (cf. la $<$ E $>$ de $\pi$ o $\tau$ E (cf. $<$ EIMI $>$ ), lo que confirma que el empleo de los dígrafos $<$ EI $>$ y $<\mathrm{OY}>$ para notar las vocales medias largas cerradas no es un hecho vinculado al empleo del alfabeto jónico o milesio.

Los demás vasos pintados del MAN que portan algún tipo de inscripción son adquisiciones de época moderna ${ }^{16}$. Por lo general, los epígrafes suelen indicar los nombres de los personajes (dioses, hombres o animales) representados en las escenas de los vasos o adjetivos referentes a estos. Otras veces se trata de la firma del

\footnotetext{
${ }^{16}$ Para la historia de la colección de vasos del MAN, que actualmente se aproxima a los dos mil ejemplares, véase en último término Cabrera, 2019.
} 
artista que ha fabricado el vaso o del pintor que lo ha decorado o incluso el nombre del propietario del vaso. Asimismo, en algún caso encontramos frases referentes a la finalidad del vaso o dirigidas a sus supuestos destinatarios. A continuación, presentamos una breve selección de diversos tipos de inscripciones, casi todas escritas en el alfabeto epicórico de Atenas.

Uno de los vasos más antiguos (575-525 a.C.) es una copa de figuras negras (MAN inv. 10947) en cuya parte exterior figura la firma del artista $\Sigma$ OK $\Lambda$ E $\Sigma$

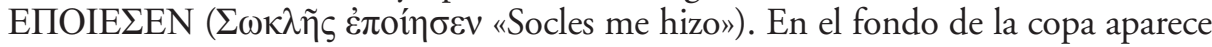
Heracles luchando contra el león y la inscripción HEP[A]K $\Lambda \mathrm{E} \Sigma\left(={ }^{\top} \mathrm{H} \rho \alpha \kappa \lambda \tilde{\eta} \varsigma\right)$.

De mediados del mismo siglo (550-500 a.C.) es una kylix de estilo ático de figuras rojas (MAN inv. 10910; CVA fasc. 2, III-I-C, 1, Pl. 2), también con la firma

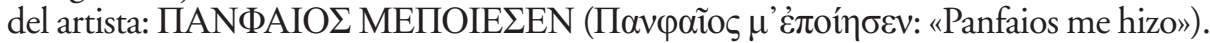

De fines del siglo VI a.C. (520-510) es una hidria de figuras negras con una escena homérica (la partida de Príamo para ir al encuentro de Aquiles y la despedida de Paris) del pintor de Príamo (MAN inv. 10920). En ella figuran varias inscripciones con los respectivos nombres de los personajes representados, algunos de ellos

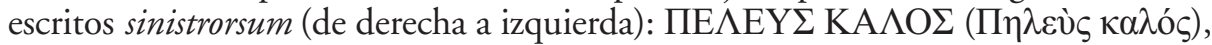

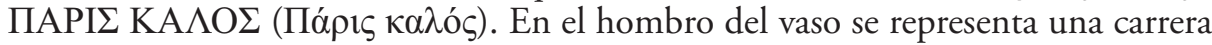
de carros, con las cuadrigas conducidas por sus aurigas y los respectivos nombres.

Aproximadamente de la misma fecha (510 a.C.) es un ánfora «bilingüe» con dos escenas (MAN inv. 11008), una con la técnica de figuras negras (Dioniso con dos sátiros y dos Ménades) y la otra en figuras rojas (Apolo, Leto, Ártemis y Ares). En

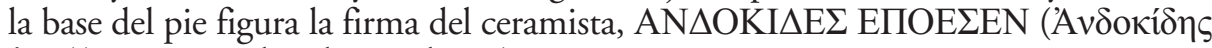
$\dot{\varepsilon} \pi \mathrm{o}(i) \eta \sigma \varepsilon v$ : «Andócides me hizo»).

Algo posterior ( 480 a.C.) es un ánfora panatenaica de figuras negras (MAN inv. 10900), en cuya cara principal aparece Atenea Prómacos avanzando de perfil entre dos columnas dóricas. A lo largo de la columna izquierda figura la inscripción,

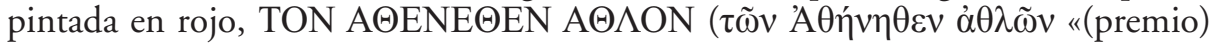
de los juegos en honor de Atenea»). En la otra cara aparece una cuadriga, indicando el tipo de competición en la que el vencedor ha ganado.

De la misma época (490-480 a.C.) es una kylix ática de figuras rojas del pintor de Antifón (MAN inv. 11269), en la que se ve a un joven atleta tras el ejerci-

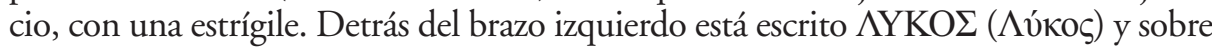
el brazo derecho, de derecha a izquierda, $\mathrm{KA} \Lambda \mathrm{O} \Sigma(\kappa \alpha \lambda \operatorname{s})$.

Poco posterior (470-460 a.C.) es una inscripción pintada en un ánfora de figuras rojas (MAN inv. 1999/99/86). En la cara A figura la inscripción KA $\Lambda O \Sigma$ ( $\kappa \alpha \lambda o ́ \varsigma$ ), referida al dios Apolo, que aparece representado tocando la lira entre Ártemis y Leto.

De mediados del siglo $V$ a.C. (450-460 a.C.) es una cratera ática de campana de figuras rojas (MAN inv. 11010), del pintor de Villa Giulia. En la escena principal aparecen Hermes, Atenea y Perseo, que se dispone a decapitar a Medusa. Tres

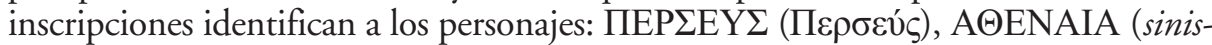
trorsum) ('A $\theta \eta v \alpha i ́ \alpha)$ y HEPME $\Sigma$ ('E $\mu$ ñ $\varsigma$, con notación de la aspiración inicial).

En la cara principal de una cratera de campana de figuras rojas de 440 a.C. (MAN inv. 1999/99/101) se representa el enfrentamiento entre dos amazonas,

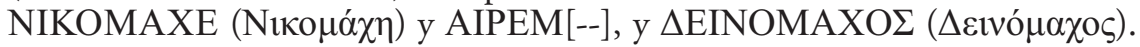


En la lécito de fondo blanco con figuras rojas del pintor de Aquiles de 440 a.C. (MAN inv. 11189) aparecen dos mujeres (¿señora y sirvienta?) haciendo los prepa-

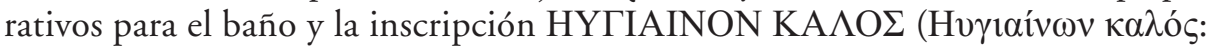
«Higienón es guapo»).

De finales del siglo $\mathrm{V}$ (420 a.C.) es una kylix ática de figuras rojas, la copa de Aison (MAN inv. 11265; CVA Madrid III-I-D, 3-4, pl. 1-5 y 15), en la que se representan las hazañas de Teseo. La firma del artista, AI $\Sigma \Omega N$ EГPAЧEN (Aï $\sigma \omega v$ Ě $\gamma \rho \alpha \psi \varepsilon v)$, está escrita ya en el alfabeto post-euclídeo, con la $\Omega$ para notar la $o$ larga abierta y $\Psi$ para /ps/. En la copa figuran más nombres, relacionados con los personajes de las diferentes escenas, sobre cuya lectura hay discrepancias entre los estudiosos

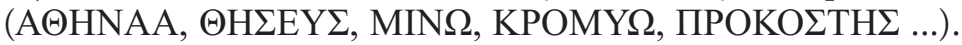

Ya del siglo IV a.C. (350-320) es una cratera de figuras rojas (MAN inv. 11094), en cuya escena principal un enloquecido Heracles se dispone a arrojar a uno de sus hijos a la pira en la que ya arden varios muebles y diversos objetos, mientras su esposa Mégara lo mira horrorizada. En una especie de galería de columnas superior asisten a la escena Alcmena, la madre de Heracles, su amigo Iolao y Manía, la locura perso-

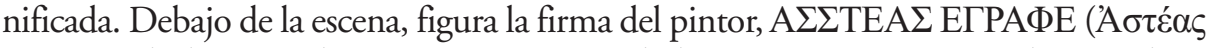
है $\gamma \rho \alpha \varphi \varepsilon:$ «lo ha pintado Asteas») y encima de los personajes aparecen los nombres

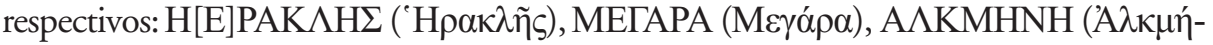
$v \eta), \operatorname{IO} \Lambda \mathrm{AO} \Sigma(\mathrm{Ió} \lambda \alpha \sigma \varsigma)$ MANIA (M $\left.\alpha v^{\prime} \alpha\right)$.

De contenido más simposíaco es la inscripción pintada en rojo, sinistrorsum, en una copa de figuras rojas (MAN inv. 11267; CVA fasc. 2, Pl. 4), en una escena

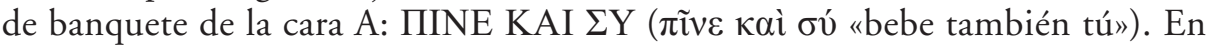
la cara B de la misma copa aparece Teseo persiguiendo al toro de Maratón, y una inscripción pintada en rojo: $\Delta \mathrm{IO} \Sigma \Pi \mathrm{HAI} \Sigma \mathrm{KA} \Lambda \mathrm{O} \Sigma$ ( $\Delta$ iòs $\pi \alpha$ ĩ $\varsigma \alpha \lambda$ ós: «El hijo de Zeus es hermoso»).

\section{LAS TRES DEDICACIONES GRIEGAS DEL MUSEO DEL PRADO}

El Museo del Prado posee una excelente colección de esculturas griegas y romanas de diferentes épocas. Esta colección, cuyo origen se remonta a la época de los Austrias, está ahora recogida en los dos tomos del excelente catálogo llevado a cabo por Stefan F. Schröder ${ }^{17}$. Son muy pocas las esculturas que portan algún epígrafe griego de época antigua; casi todas las inscripciones griegas son de época moderna (ver infra \$7).

${ }^{17}$ A partir de ahora $C E C M P$ (para la referencia completa, véase la bibliografía final). De los 89 retratos incluidos en el primer tomo (1993), sólo 19 son griegos, el resto son romanos. El segundo tomo del catálogo (números 90 a 219) está dedicado a las esculturas mitológicas. 
5.1. Dedicación a Tito Estatilio (Prado inv. 18-E; CECMP I, 49, con fotos del busto y de la inscripción; $I G$ XIV 1087, IGUR II 951). Encontrada en Roma. El pie del busto de T. Statilio con la dedicación antigua a este personaje, de la primera mitad del siglo II d.C., se ha unido a un busto moderno del siglo XVI.

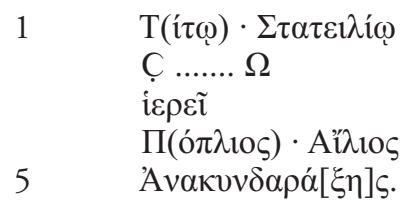

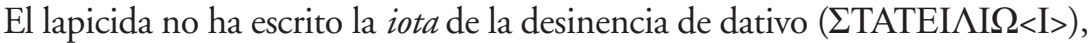
algo muy frecuente en esta época, tras perderse el segundo elemento de los diptongos largos. Según Schröder (CECMP I, 180), Tito Statilio, a quien estaba dedicado el busto originario, fue probablemente un sacerdote, un liberto procedente de la parte oriental del Imperio Romano. El donante del busto, Publio Elio Anakyndaraxes, era un romano de origen persa manumitido bajo el emperador Adriano.

5.2. Dedicación de Publio Licinio Prisco a Poseidón (Prado inv. 3-E; CECMP II, 193 A-B, con foto del grupo [lámina 25] y de la inscripción [fig. 90]; IG IV 202; $I G$ XIV 2543; SEG 53.283; SEG 54.1008). Dedicación inscrita sobre la cabeza de un delfín que forma parte de un grupo escultórico que representa a Poseidón (193 A), fechado en 130-140 d.C. (según criterios estilísticos) ${ }^{18}$. La escultura procede de Roma y probablemente fue hallada en Corinto o en Istmia. La ficha de la inscripción (193 B) corre a cargo de Jaime Curbera, quien hace un minucioso estudio del epígrafe. La segunda parte de la línea 3, así como la línea 4, se deben a Curbera. La inscripción contiene una dedicación a Poseidón por Publio Licinio Prisco, a quien se identifica con Publio Licinio Prisco Juventiano ${ }^{19}$.

$$
\begin{aligned}
& \Pi(o ́ \pi \lambda \text { เos }) \cdot \Lambda \text { ı́́vios } \\
& \text { Прєі̃бко }
\end{aligned}
$$

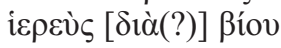

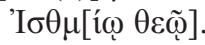

5.3. Dedicación a Neón (Prado inv. 327-E; CECMP i, 77, con fotos del busto y de la inscripción; $I G$ XIV 2544). Se trata del busto de un joven, de procedencia desconocida, quizás de Asia Menor. Fechado por Schröder en 200-210 d.C. (según

\footnotetext{
${ }^{18}$ Números 193 A (escultura) y 193 B (dedicación) del catálogo.

${ }^{19}$ Véanse los argumentos de J. Curbera loc. cit., 422.
} 
criterios iconográficos). En el pedestal figura el nombre del personaje en dativo:

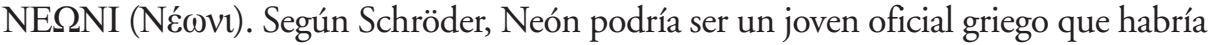
muerto en una de las campañas en la frontera oriental del Imperio.

\section{LAS MONEDAS GRIEGAS}

Aunque generalmente se considera que su estudio corresponde más bien a la numismática, no podemos dejar de mencionar aquí algunas colecciones de monedas griegas existentes en la CAM, muchas de las cuales presentan interesantes leyendas en griego. Citemos la excelente colección de monedas griegas de la Casa de la Moneda de Madrid, que cuenta con más de 900 ejemplares, los más antiguos de los siglos VIIVI a.C., procedentes de todo el territorio griego y sus áreas de influencia ${ }^{20}$. Asimismo, es digna de mención la colección de aproximadamente mil monedas griegas de la Real Academia de la Historia, clasificadas y publicadas el año 2006 por Ana Vico en un excelente catálogo (véase la bibliografía final), así como las colecciones del Museo del Prado, del Gabinete Numismático del Museo Arqueológico Nacional y del Museo Cerralbo $^{21}$.

\section{LAS INSCRIPCIONES DE ÉPOCA MODERNA: LA COLECCIÓN AZARA}

Entre las múltiples actividades llevadas a cabo por José Nicolás de Azara durante su estancia en Roma como diplomático, se encuentra la de arqueólogo. Fruto de sus excavaciones desde 1779 en la Villa de los Pisones, cerca de Tívoli, son los quince retratos de filósofos y autores griegos, origen de una excelente colección que luego completará con la adquisición de numerosas obras más. Menos el retrato de Alejandro Magno, que Azara regaló a Napoleón y que actualmente se encuentra en el Louvre, el resto de los retratos, junto con otras esculturas, fueron donados al rey Carlos IV.

${ }^{20} \mathrm{Cf}$. Encinas Bodega, 2019. Citemos, por ejemplo, una dracma procedente de Rosas (Girona),

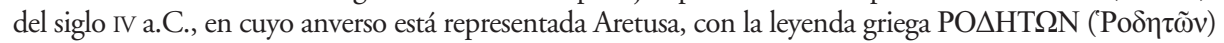
a su izquierda.

${ }^{21}$ Para la colección numismática del MAN, véase Otero Morán, 2016 y 2019. El catálogo puede consultarse en http://www.man.es/man/coleccion/catalogo-cronologico/numismatica.html\#accesocata-

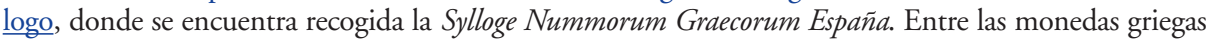
de este museo destaca la octodracma de oro (inv. 2016/31/1), fechada entre 246-221 a.C. y perteneciente a las emisiones acuñadas con el nombre de Arsínoe II (AP $\Sigma$ INOH $\Phi \mathrm{I} \Lambda \mathrm{A} \Delta \mathrm{E} \Lambda \Phi O \Sigma$ ), aunque en ella aparece representada Berenice II, esposa de Ptolomeo III. Para la colección numismática del Museo Cerralbo, cf. https://www.culturaydeporte.gob.es/mcerralbo/coleccion/coleccion0/monedas.html. Asimismo, pueden consultarse también las bases de datos recogidas en la nota 1. 
Dieciséis de los retratos se encuentran actualmente en la Casa del Labrador del Palacio de Aranjuez (Galería de las Estatuas) ${ }^{22}$, el resto en el Museo del Prado. Casi todas las cabezas son copias romanas de originales griegos.

Tanto las cabezas procedentes de la excavación en Tívoli, como las adquiridas por Azara posteriormente, fueron restauradas y montadas sobre bustos de mármol en forma de herma. En uno de los laterales de los hermas de las cabezas halladas en Tívoli Azara hizo grabar una inscripción latina con su nombre y los datos de la excavación ${ }^{23}$.

SIGNVM IN TIBVRTINO PISONVM EFFOSSVM ANNO MDCCLXXIX IOS. NIC. AZARA REST. C. (= RESTAVRANDVM CVRAVIT)

«Retrato excavado en el año 1779 en la Villa de los Pisones y hecho restaurar por Jos. Nic. Azara»

Asimismo, en los hermas de la mayoría de los retratos, tanto de los hallados en Tívoli como de los adquiridos posteriormente, Azara hizo inscribir leyendas en griego con el propósito de identificar a los personajes representados. Los nombres grabados no siempre corresponden correctamente al personaje retratado. En efecto, la mayoría de las veces las identificaciones son erróneas y, además, algunas de ellas presentan faltas de ortografía ${ }^{24}$. A continuación, vamos a hacer un breve repaso de los hermas que llevan inscripción griega identificativa.

De los retratos conservados en el Prado sólo cuatro llevan las dos inscripciones, la griega identificativa y la latina que confirma que proceden de la excavación de Tívoli.

CECMP I, 4 (Prado inv. 23-E. Tívoli, Villa de los Pisones) es una copia romana de un retrato de Milcíades de ca. 340 a.C. La identificación de Azara con el filósofo Zenón es errónea. En la Galería de Estatuas de Aranjuez hay otra cabeza de la colección Azara igual a la del Prado, hallada también en las excavaciones de Tívoli. Sorprende que, a pesar de que es evidente que ambas cabezas corresponden al mismo personaje, Azara añadiera en cada una un nombre diferente: en la del Prado ZHN $\Omega N$

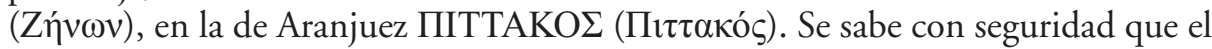
personaje representado en ambos retratos es el estratego Milcíades, como muestra la cabeza conservada en Rávena, igual a los dos retratos de Azara, con la inscripción identificativa correspondiente.

${ }^{22}$ Aunque sólo diez de ellos presentan la característica inscripción en latín con el nombre de Azara y los datos del hallazgo, se piensa que los dieciséis bustos proceden de la colección de Azara (cf. Elvira Barba, 1993: 125).

${ }^{23} \mathrm{El}$ texto es prácticamente el mismo en todas las inscripciones, salvo algunas insignificantes diferencias, generalmente debidas a errores o lapsus o a las diferentes variantes en que aparece el nombre de Azara.

${ }^{24}$ Algunas de estas faltas fueron subsanadas en los grabados que acompañaban a la edición española que Azara hizo de la Historia de Marco Tulio Cicerón, de C. Middleton. 
CECMP I, 13 (Prado inv. 19-E. Tívoli, Villa de los Pisones) es una réplica romana del segundo cuarto del siglo II d.C. de un retrato del filósofo Hermarco de Mitilene (325-260/250 a.C.), sucesor de Epicuro, al que Azara identificó errónea-

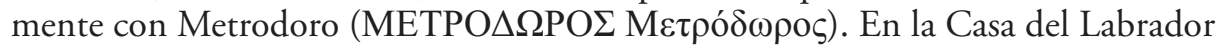
de Aranjuez hay un busto de Metrodoro (cf. infra no 10 de Aranjuez), que asimismo Azara consideró de manera errada que era Epicuro, cuyo nombre añadió al busto.

Se han hecho varias propuestas sobre la identidad del personaje representado en CECMP I, 14 (Prado inv. 13-E. Tívoli, Villa de los Pisones), copia romana de comienzos del siglo II d.C. de un retrato de $c a .280$ a.C., identificado sin razón con

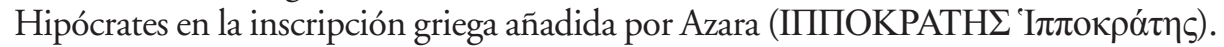

El cuarto retrato del Prado que presenta también la inscripción latina además de la griega, CECMP II, 92 (Prado inv. 78-E. Tívoli, Villa de los Pisones), es una réplica de una copia romana, probablemente del siglo I d.C., que reproduce la cabeza de la estatua de Aristogitón de un grupo escultórico en bronce de los Tiranicidas de $c a .477$ a.C., hecho para sustituir al primitivo grupo robado por los persas ${ }^{25}$. La inscripción griega añadida por Azara lo identifica con el filósofo Ferécides $\Phi E P E K Y \Delta H \Sigma(\Phi \varepsilon \rho \varepsilon \kappa v ́ \delta \eta \varsigma)$. Es muy probable que el fuste correspondiente a esta cabeza, actualmente perdido, fuera el que en el siglo XV se encontraba en la iglesia

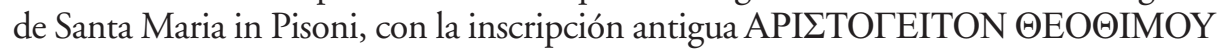

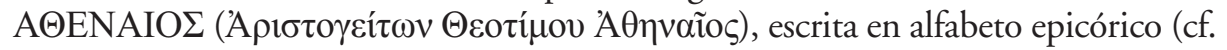
Blanco - Lorente, 1981: 55).

Los restantes retratos de la Colección Azara conservados en el Prado no llevan la inscripción latina, por lo que deben de pertenecer a los adquiridos posteriormente por el diplomático.

CECMP I, 7 (Prado inv. 81-E) es una copia romana de un retrato de Menandro de ca. 290 a.C., atribuido por la inscripción griega a Aristóteles (API $\Sigma T O T E \Lambda H \Sigma$

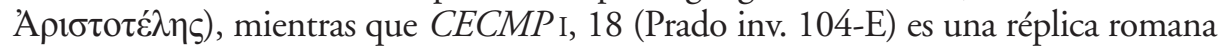

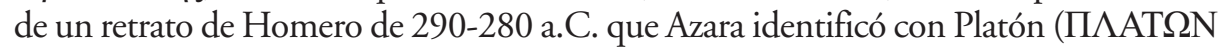

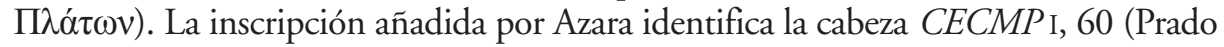
inv. 79-E), del siglo II d.C., con Bías de Priene, filósofo del siglo VI a. C y uno de los Siete Sabios (BIA $\Sigma$ Bías). De los dos últimos retratos que no llevan inscripción latina, $C E C M P$ II, 100 (Prado inv. 55-E) es una réplica romana (siglo I d.C.) de un herma arcaizante ático de 425-400 a.C. Influido probablemente por el parecido del retrato con la representación de Dioniso a partir de la segunda mitad del siglo IV, el tipo llamado Sardanápalo, Azara lo bautizó con este nombre. La inscripción presenta, además, algunas faltas de ortografía ( $\Sigma \mathrm{AP} \triangle \mathrm{ANA \Pi A} \Lambda \Lambda \Omega \Sigma$ sic $=\Sigma \alpha \rho \delta \alpha \nu \alpha ́ \pi \alpha \lambda \mathrm{s})$. Por último, CECMP II, 101 (Prado inv. 15-E) es igualmente una réplica romana,

${ }^{25}$ Para más detalles sobre los dos grupos escultóricos de los tiranicidas, las dos estatuas de mármol de Nápoles y la réplica del Prado, véase CECMP II 92. 
de mediados del siglo II d.C., de un herma griego de 410-400 a.C. Hasta fines del siglo $\mathrm{V}$ a.C. todos los hermas representaban a Hermes, por lo que probablemente sea este el dios allí representado, aunque la inscripción griega de Azara lo identifica erróneamente con el cómico Aristófanes (API $\Sigma T O \Phi A N H \Sigma$ A

En lo que respecta a los retratos de Aranjuez, en diez de ellos figuran las dos inscripciones, la latina que confirma su procedencia de Tívoli y la griega identificativa (números 3, 4, 5, 6, 7, 10,11,13,14 y 15), en otros tres sólo la inscripción griega ( 1,2 y 16), mientras que en los tres restantes $(8,9$ y 12) no hay ninguna inscripción. Como hemos apuntado supra, se admite generalmente que los dieciséis retratos proceden de la Colección de Azara, aunque hay discrepancias sobre la autenticidad de algunos de los que no presentan la inscripción latina (¿obras del s. XVIII?). Para las referencias seguimos la numeración de Hertel (1983 y 1985), seguida a su vez por Elvira Barba (1993 y 1994).

La cabeza del herma 3 de Aranjuez (Tívoli, Villa de los Pisones), copia romana de un original del siglo IV a.C., fue identificada por Azara como la del héroe Heracles (HPAK $\Lambda \mathrm{H} \Sigma{ }^{\circ} \mathrm{H} \rho \alpha \kappa \lambda \tilde{\eta} \varsigma$ ), interpretación considerada generalmente como correcta (cf. Elvira Barba, 1994: 63). El número 4 (Tívoli, Villa de los Pisones) es una copia de época imperial de un retrato de Milcíades del siglo IV a. C, identificado erróneamente por Azara como Pítaco de Mitilene, uno de los Siete Sabios de Grecia

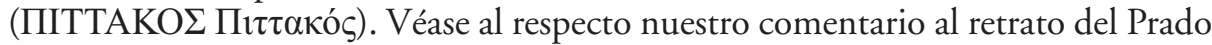
CECMP I, 4 (inv. 23-E).

El número 5 (Tívoli, Villa de los Pisones), copia de un original del siglo III a.C.,

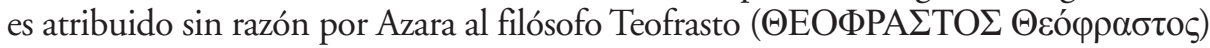
y el número 6 (Tívoli, Villa de los Pisones), copia de un original del siglo $\mathrm{V}$, al histo-

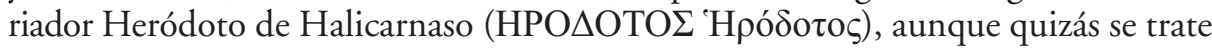
de Aristogitón.

La cabeza del herma 7 (Tívoli, Villa de los Pisones) es copia de un original del siglo IV, atribuido por Azara a Sófocles ( $\Sigma \mathrm{O} \Phi О \mathrm{~K} \Lambda \mathrm{H} \Sigma \Sigma$ Бофок $\lambda \tilde{\eta} \varsigma)$, mientras que el número 10, también hallado en la Villa de los Pisones, es una copia de época imperial de un retrato del siglo III a.C., atribuido erróneamente a Epicuro (ЕПIКОYРО $\Sigma$

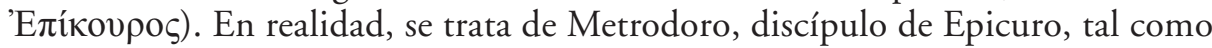
muestra un herma doble del Museo Capitolino con los retratos de ambos, con inscripciones identificativas (cf. Elvira Barba, 1994: 63).

La cabeza 11 de Aranjuez (Tívoli, Villa de los Pisones), copia romana de un retrato del siglo II a.C., corresponde, según Azara, al filósofo Carnéades (KAPNEA $\triangle \mathrm{H} \Sigma$

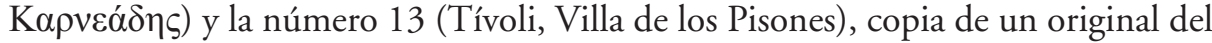
siglo III a.C., a Sócrates ( $\Sigma \Omega$ KPATH $\Sigma \Sigma \omega \kappa \rho \alpha ́ t \eta \varsigma)$, en ambos casos probablemente de manera acertada (cf. Elvira Barba, 1994: 64).

Los retratos 14 y 15 (Tívoli, Villa de los Pisones) son copias de originales del siglo IV a.C. Sin ninguna base una vez más Azara consideró que la primera cabe-

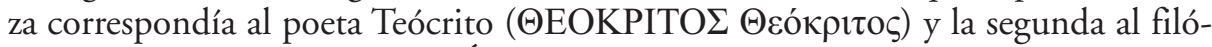

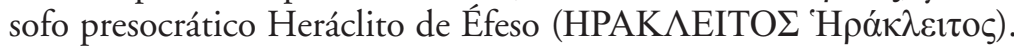

Como hemos indicado supra, los retratos 1, 2 y 16 de Aranjuez no llevan la consabida inscripción latina referente a la excavación de Tívoli, por lo que deben de ser obras adquiridas posteriormente por Azara. Probablemente por esa razón 
Hertel los considera obras de época moderna del siglo XVIII, así como los retratos 8,9 y 12, que no llevan ninguna inscripción, ni griega ni latina. El personaje del herma 1 es identificado en la inscripción de Azara como Periandro, tirano de Corinto

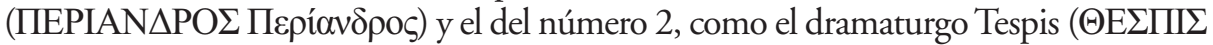

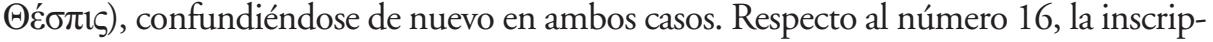
ción de Azara identifica este retrato con el orador Demóstenes, al parecer correcta-

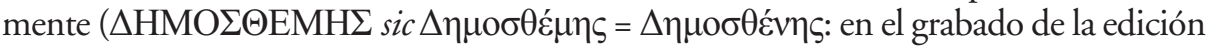
de la vida de Cicerón, aparece la forma correcta $\triangle \mathrm{HMO} \Sigma \Theta E N H \Sigma$ ).

Como se ha podido ver a lo largo de estas líneas, aunque el patrimonio epigráfico griego de la Comunidad de Madrid no es muy abundante, sus inscripciones constituyen una buena muestra de los numerosos avatares que desde la Antigüedad han experimentado estos documentos epigráficos y de cómo han terminado enriqueciendo los fondos de importantes museos y colecciones nacionales. Como decíamos al comienzo de nuestro trabajo, hasta la actualidad no se disponía de un estudio de conjunto, aunque sí de excelentes corpora y estudios monográficos, sobre los diferentes textos epigráficos (grafitos, inscripciones, monedas, vasos, esculturas) que de un modo y otro han pasado a formar parte de los fondos de esta comunidad autónoma. Nuestro propósito en este homenaje al profesor Ángel Martínez Fernández ha sido colmar de alguna manera esa laguna.

\section{REFERENCIAS BIBLIOGRÁFICAS}

Almagro-Gorbea, M. (2019): «Objetos griegos del Gabinete de Antigüedades de la Real Academia de la Historia (Madrid)», en X. AQuiluÉ - P. Cabrera (coords.), Iberia Graeca. Arte griego en los museos y colecciones de la península Ibérica, Girona, pp. 67-78.

AQuilué, X. - CABRERA, P. (coords.) (2019): Iberia Graeca. Arte griego en los museos y colecciones de la península Ibérica, Girona.

Blanco, A. - Lorente, M. (1981): Catálogo de la Escultura, Museo del Prado, Madrid.

BRIXHE, C. (1987): Essai sur le grec anatolien au début de notre ère, Nancy.

BRIXHE, C. (1991): «Étymologie populaire et onomastique en pays bilingue», RPh, 65/1: 67-81.

CABrera, P. (2019): «Objetos griegos del museo arqueológico nacional (Madrid). Las coleccciones de antigüedades griegas», en X. AQUiluÉ - P. CABrera (coords.), Iberia Graeca. Arte griego en los museos y colecciones de la península Ibérica, Girona, pp. 25-36.

CECMP I = Schröder, 1993.

CECMP II = Schröder, 2004.

$C V A=$ Mélida, 1930 .

Elvira Barba, M. A. (1993): «La actividad arqueológica de D. Nicolás de Azara», en F. GASCó La CALle - J. L. Beltrán - J. T. SARACHO Villalobos (eds.), La Antigüedad como argumento: historiografia de arqueología e historia antigua en Andalucía, pp. 125-152.

ElVIRA BARBA, M. A. (1994): «La actividad arqueológica de D. José Nicolás de Azara», Reales Sitios: Revista del Patrimonio Nacional 122: 57-65. 
Elvira Barba, M. A. (1995): «El arte antiguo en la Casa del Labrador de Aranjuez», Boletín de la Asociación Española de Amigos de la Arqueología XXXV: 217-228 (Madrid).

Elvira Barba, M. A. (2008): «La colección de escultura de la Casa Real Española durante el siglo XVIII», en S. F. SCHRÖDER (coord.), Entre dioses y hombres: esculturas clásicas del Albertinum de Dresde y el Museo del Prado, pp. 122-136.

EnCINAS BodegA, I. (2019): «Moneda griega en el Museo Casa de la Moneda (Madrid)», en X. AQuilué - P. Cabrera (coords.), Iberia Graeca. Arte griego en los museos y colecciones de la península Ibérica, pp. 57-66.

FEISSEL, D. (1982): «Remarques de toponymie syrienne d'après des inscriptions grecques chrétiennes trouvées hors de Syrie», Syria 59 (3-4): 319-343.

GIGNAC, F. Th. (1976): A Grammar of the Greek papyri of the Roman and Byzantine periods, vol. I: Phonology, Milán.

Gujjarro Ruano, P. (2016), «De Hoz Bravo, Ma Paz, Inscripciones griegas de España y Portugal. Bibliotheca Archaeologica Hispana 40, Madrid, Real Academia de la Historia, 2014, 612 pp.», Emerita LXXXIV: 177-202.

Hertel, D. (1983): «Los bustos de emperadores romanos, las estatuas ideales de yeso y los retratos griegos de la Casa del Labrador de Aranjuez», Reales Sitios: Revista del Patrimonio Nacional 78: 17-36.

Hertel, D. (1985): «Die griechischen Porträts der Sammlung Azara und ihre Rezeption in der Casa del Labrador von Aranjuez», Madrider Mitteilungen 26: 234-242.

Hoz, M. P. DE (2014): Inscripciones griegas de España y Portugal, Madrid.

$I G E P=$ de Hoz, 2014.

Melchert, H. C. (2013): «Naming Practices in $2^{\text {nd }}$ and $1^{\text {st }}$ Millennium Western Anatolia», en R. PARKer (ed.), Personal Names in Ancient Anatolian, Oxford.

Mélida, J. R. (1930): Corpus vasorum antiquorum. Espagne. Fasc. 1, Madrid, Musée Archéologique National, Madrid.

Otero, P. (2016): «Un nuevo museo para una antigua colección. Monedas, medallas y "otras curiosidades" en el renovado Museo Arqueológico Nacional», en XV Congreso Nacional de Numismática (Madrid, 28-30 octubre 2014), pp. 27-60.

Otero, P. (2019): «Monedas griegas del Museo Arqueológico Nacional (Madrid). El Gabinete Numismático", en X. AQUilué - P. CABRERA (coords.), Iberia Graeca. Arte griego en los museos y colecciones de la peninsula Ibérica, Girona, pp. 37-46.

Ramírez SÁdABA, J.-L - Mateos CruZ, P. (2000): «Catálogo de las inscripciones cristianas de Mérida», Cuadernos emeritenses 16: 7-323.

Ramírez SÁnCheZ, M. (2015): «Inscripciones griegas de España y Portugal (IGEP)» E-pigraphia: Epigrafia en Internet, 8 diciembre 2015 [https://epigraphia.hypotheses.org/431. Consulta: 22 noviembre 2020].

ROBERT, L. (1945): Le sanctuaire de Sinuri près de Mylasa, I. Les inscriptions, Mémoires de l'Institut français d'archéologie de Stamboul 7, Paris.

Robert, L. (1963): Noms indigènes dans l'Asie Mineure gréco-romaine, Paris.

Robert, L. - RoberT, J. (1944): «Bullétin épigraphique», REG 57: 175-241.

Sarrazanas, Cl. (2016): «María Paz DE HOZ, Inscripciones griegas de España y Portugal (IGEP). Madrid, Real Academia de la Historia, 2014. 1 vol. 612 p.», L'Antiquité Classique 85: 429-431. 
SCHRÖDER, S. F. (1993): Catálogo de la escultura clásica del Museo del Prado. Retratos, Museo del Prado, vol. I, Madrid.

SCHrÖDER, S. F. (2004): Catálogo de la escultura clásica del Museo del Prado, Escultura mitológica, Museo del Prado, vol. II, Madrid.

SCHRÖDER, S. F. (2019): «Esculturas griegas en el Museo Nacional del Prado (Madrid)», en X. AQuilué - P. CABrera (coords.), Iberia Graeca. Arte griego en los museos y colecciones de la península Ibérica, Girona, pp. 47-56.

Sinuri $=$ Robert, 1945 .

Vernet, M. (2012-2014): «The Etymology of the PNs Artimas and Arteimas from Asia Minor: a New Explanation", Faventia 34-36: 99-110.

Vico Belmonte, A. (2006): Catálogo de monedas griegas de la Real Academia de la Historia, Madrid. Zgusta, L. (1964): Kleinasiatische Personennamen, Praga. 